\title{
Comparative study for connecting new flare capacity to existing flare Systems
}

\author{
Mohamed Shamandy Fouad ${ }^{\mathrm{a}}$, Walaa Mahmoud Shehata ${ }^{\mathrm{b}}$, Fatma Khalifa Gad ${ }^{\mathrm{b}}$, Nabil Abdelsadek \\ Abdelaleem $^{c}$ \\ ${ }^{a}$ KHALDA Petroleum Company, Maadi, Egypt

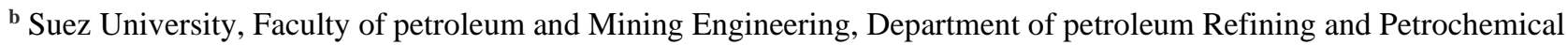 \\ Engineering. \\ ${ }^{\mathrm{c}}$ GENERAL Petroleum Company, Nasr City, Egypt
}

\begin{abstract}
Process facility operations are equipped with flare system to dispose flammable, toxic, or corrosive vapors to an environmentally acceptable gas for release to the atmosphere from both normal operational venting and relief during abnormal conditions. For safe incineration and radiation considerations flares are located at a remote point from the plant. Flare system when designed is to be limited for a certain capacity for a relieved gas; in addition, it may be designed in excess for future process facilities which may be further added to an existing one.

KHALDA Petroleum Company is an owner company of oil and gas plants in Egypt. The company started a remote facility in 2005 named as "QASR start of line" which equipped with a limited flaring system capacity of $416,800 \mathrm{~kg} / \mathrm{hr}$ for each flare related to phases PH-1 and PH-2. After 10 years of operation the wells depletion occurred which affected the production capacity and hence the company decided to maintain the productivity. A new compression project is designed to improve recovery as the reservoir production rate and pressure decline. The new compression project facilities vent and disposal need a flare system in case of the emergency. This paper discusses two different scenarios available for connecting the new compression project facilities vent and disposal system either to the existing flare systems or to another destination, which better, safer and more economic
\end{abstract}

Keywords

Flaring system; depressurization; emergency shutdown; conventional blowdown; sequential blowdown; maximizing relieving capacity.

Article Received: 18 October 2020, Revised: 3 November 2020, Accepted: 24 December 2020

\section{Introduction}

In most process facility operations gas and vapor have to be disposed of safely, quickly, and without environmental impact [1]. When the venting gas or vapor cannot be converted to useful energy it is routed to a remote point for safe incineration, which is called faring. Flares are the most economical and customary means of disposing of excess light combustible gases in the process industries. The primary function of a flare is to convert flammable, toxic, or corrosive vapors to an environmentally acceptable gas. These acceptable gases are released to the atmosphere from both normal operational venting and relief during abnormal conditions [1,2].
Flare is the last line of defense in the safe emergency release system in the petroleum and chemical plants. The flare provides a means of safe disposal of the vapor streams from its facilities, by burning them under controlled conditions. Such that adjacent equipment or personnel are not exposed to hazards, and at the same time obeying the environmental regulation of pollution control and public relations requirements. The chemical process used for flaring is a high temperature oxidation reaction to burn combustible components, mostly hydrocarbons, or waste gases from industrial operations [3].

In combustion, the gaseous hydrocarbon (natural gas, propane, ethylene, propylene, butadiene, 
butane and etc.) reacts with atmospheric oxygen to form carbon dioxide $\left(\mathrm{Co}_{2}\right)$ and water. Several byproducts formed will be carbon monoxide, hydrogen and others dependent upon what is being burned. Efficiency of hydrocarbon conversion is generally over $98 \%$ [4-8].

The flare system must be correctly sized to handle the material vented during depressuring, especially peak flow. During the blowdown operation, a large amount of material must be disposed of simultaneously. It is necessary to ensure that there is sufficient flare discharge capacity to do this without violating hydraulic constraints in the flare piping. This causes overpressures or excessive vibrations or exceeding radiation limits from the flare tip. It is also necessary to accurately determine what size of restriction orifices or other flow-controlling devices and flare connections are required for depressuring each section of the plant [9-11].

Calculations of depressurization system reveal whether high volumes of gases will be flowing through the pipe header to the flare. In some cases, simultaneously depressurizing all the process and equipment, vessels, and piping in a plant will be difficult to accomplish (due to pipe sizing and economic impacts). In these cases, sequential or segmented depressurization of vessels should be considered. Providing for the worst vessels first or controlling the system to depressurize the area most affected first are possible scenarios that can be employed [11-14].

\section{Case study}

Qasr is a large, normally pressured gas-condensate reservoir located in the Western Desert of Egypt approximately $525 \mathrm{~km}$ west of Cairo. The field is operated by Khalda Petroleum Company (KPC), a joint venture between Apache Corporation and Egyptian General Petroleum Company (EGPC).

Field production is initially handled at the Start of line (SOL) Qasr Plant. The Qasr gas and condensate free flows from the wellheads through the Qasr Phase I and Phase II facilities and export pipelines to the SHAMS manifold and Salam gas plant under reservoir pressure until year 2015. As the reservoir pressure declines the peak gas rate of $800 \mathrm{mmscfd}$ are no longer be achievable. The Qasr Compression Project is designed to improve recovery as the reservoir production rate and pressure decline.

There are two existing independent HP flare systems, one for Phase I facilities and the other for Phase II facilities. Phase I HP flare system, comprising a duplex stainless steel collection system, carbon steel KO drum, carbon steel riser and stack on which is mounted a sonic flare tip with design capacity of $416,800 \mathrm{~kg} / \mathrm{h}$. Phase II HP flare system, comprising a duplex stainless steel Pipe collection system, carbon steel KO drum, carbon steel riser and stack on which is mounted a sonic flare tip with design capacity of $416,800 \mathrm{~kg} / \mathrm{h}$.

This paper discusses two options suggested when connecting a new facility to an existing process plant considering tie-in both flares systems into the existing.

The available options for Flare tie-in are sequenced flare to $\mathrm{PH}-1$ or $\mathrm{PH}-2$ existing, flare combination to both $\mathrm{PH}-1$ and/ or PH-2 and independent new compression flare utility. These options are competitive to find the feasible method for atmospheric disposal of the compression area (gas compressors and condensate suction drum) to HP flare blowdown loads to achieve the following objectives: -

- Overpressure protection for process and utility systems

- Means of automatic depressurizing of the Compression Project process facilities during emergency or upset conditions. 
- Safe disposal of hydrocarbons which may from time to time - be vented from the compression plant during abnormal operations and plant maintenance, start-up or shutdown.

- Allow controlled manual depressurization of equipment items for maintenance, inspection and testing.

- Minimize the potential pollution of the environment.

These tie-in options will compatible with one of the following blowdown scenarios:

Conventional blowdown "Total plant
blowdown starting from max. operating
pressure reaching to 7 barg within 15 min".

Sequential blowdown starting from max. operating pressure reaching to 7 barg within 15 min., applying the sequential blowdown philosophy between Phase 1, Phase 2 and

\section{Compression areas.}

The plant layout is arranged into individual sections can be considered as separate fire zones. A credit will be taken for the area where the highest risk is occurred to be depressurized firstly then after the area under lower risk. This allows one section to be affected by a fire, without their necessarily being an impact on other sections.

Phase- 1 process facility consists of 12 BDVs used in between the emergency shutdown valves dedicated to be used in emergency shutdown to release the process plant facility hazardous hydrocarbon.

Phase-2 process facility consists of 7 BDVs used in between the emergency shutdown valves dedicated to be used in emergency shutdown to release the process plant facility hazardous hydrocarbon.

The process facility emergency shutdown valves and blowdown valves is described in Figure 1 for Phase- 1 and Figure 2 for Phase-2.

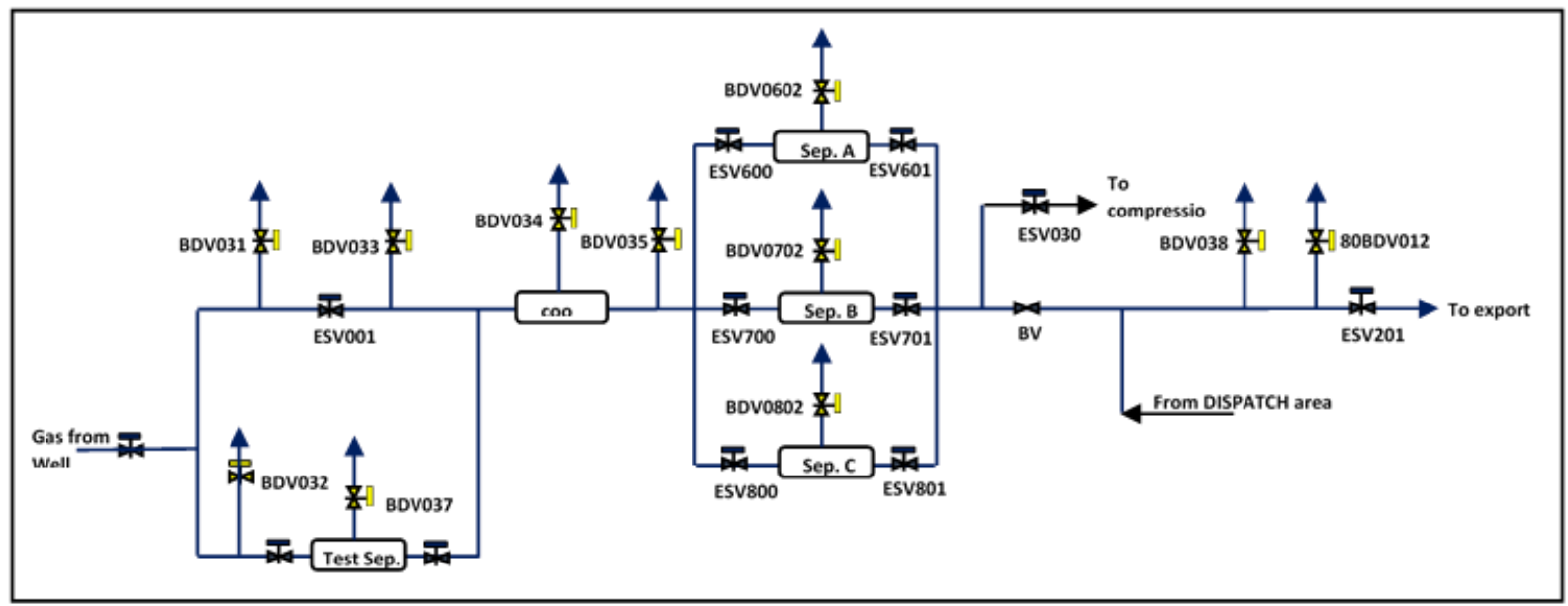

Figure 1: process flow diagram for Phase-1 


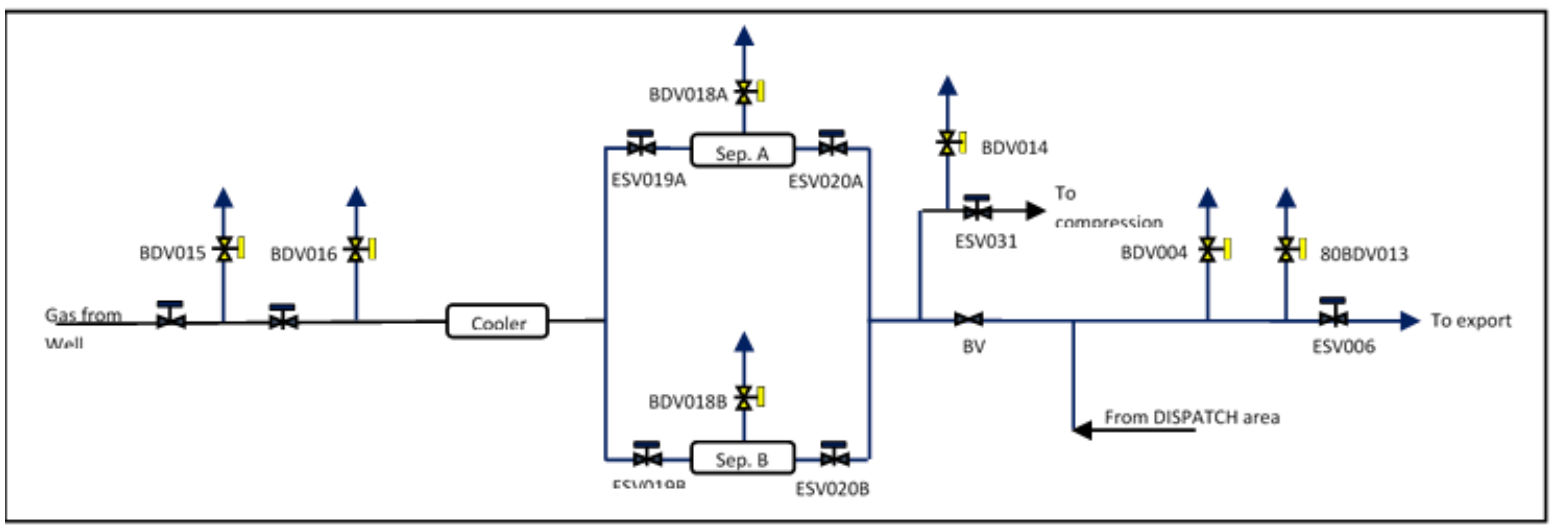

Figure 2: Process flow diagram for Phase-2

Compression facilities are new facilities added to the existing process facilities (Phase- 1 and Phase-2 facilities) to increase the system pressure. These facilities are collected in a compression unit (Figure 4, a), a condensate handling system (Figure 4, b) and fuel system (Figure 4, c). The condensate system consists of two identical suction drums A (cond. Drum A) and B (cond. Drum B) units, each in equal capacities and used in $100 \%$ redundancy as one in service and the second one is standby which is assumed to be depressurized and under positive nitrogen pressure to be preserved free of hydrocarbon liquid and gases. The fuel system contains a scrubber to free the gas from any entrained liquids associated with the gas. Also, there are preheaters to raise the gas stream temperature in a preparation step for conditioning the gas to be subjected to pressure reduction without hydrate formation.

The new compression unit facility consists of 12 BDVs. These valves are used in between the emergency shutdown valves to release the process plant facility hazardous hydrocarbon.

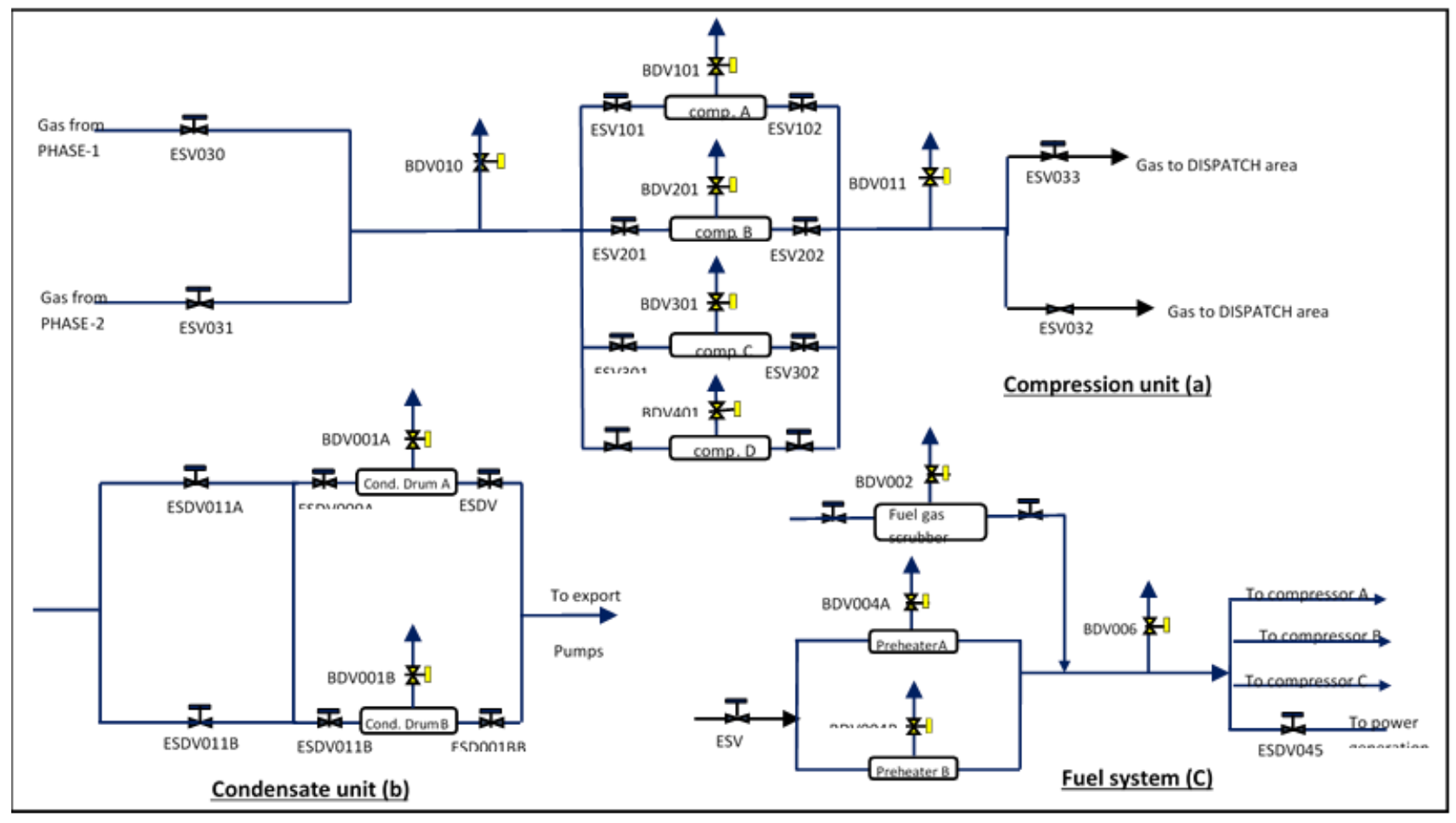

Figure 3: Compression unit facilities 


\section{Methodology}

Scenario-1: Implementation an independent new Compression Flare system

The blowdown philosophy complies with the API 521 standards to release the hazardous hydrocarbon gas from the maximum pressure at 131 barg during the emergency to be reduced in 15 minutes to reach 7 barg. In such case the process facility can be maintained safe in case of emergency fire or even in the preliminary gas release.

In this suggested scenario, a new independent compression flare system will be constructed. The compression initial pressure assumed to be the maximum operating pressure for all equipment. As a result of pressure reduction at the compression suction header, the depressuring pressure will vary according to the operating condition. The blowdown philosophy applied to phase-1 and phase-2 is also applied to the new compression flare system.

Scenario-2: Sequenced Flare to Phase-1 and Phase-2 existing

This scenario is based on arranging the plant flare systems into individual sections. These sections can be considered as separate fire zones. This allows one section to be affected by a fire, without an impact on the other sections. This design limits the requirement of full plant blowdown, which is desirable because an emergency blowdown results in the loss of a significant mass of hydrocarbons to the atmosphere (more conservative for $\mathrm{HC}$ gas). This sequenced scenario depends on relieving the compression facilities to phase- 1 or phase- 2 flare system after the piping and equipment of phase- 1 or phase- 2 have reached to 7 barg at 15 minutes. Connection of compression facilities to phase- 1 or phase-2 flare system depends on the design capacity of the flaring system $(416,800 \mathrm{~kg} / \mathrm{h})$.

\section{Results and discussion}

The simulation is built using Aspen HYSYS program to perform the process plant facility blowdown scenarios in case of fire. The process plant facilities are simulated using Aspen Tech process modeling HYSYS V.10 and corresponding Aspen Flare System Analyzer V 10 for simulating the relieved gas different scenarios. The fire scenario is based on API-521 standard fire case. Each individual process equipment is to be sized according to the mechanical data sheet. Each pipe is to be specified according to the metal type, schedule and the nominal diameter and hence to state the pipeline length to determine the proposed volume of that pipe.

Scenario-1: Implementation an independent new Compression Flare system

The simulation is built using Aspen HYSYS v. 10 to perform the process plant facility blowdown scenario in case of fire through phase- 1 and phase2 facilities. The fire scenario is based on API-521 standard fire case. Total flared gas during blowdown equals the sum of all BDVs Blowdown valves individually in each phase. All system depressuring in the moment of emergency case activation are taken into consideration to achieve the depressuring through 15 minutes. The total peak flow calculated for phase- 1 is illustrated in Table 1 and for phase- 2 is illustrated in Table-2:

Table 1: Total flared gas during blowdown for phase 1 (scenario-1) 


\begin{tabular}{|c|c|c|c|c|c|c|c|c|}
\hline \multirow{2}{*}{$\begin{array}{c}\text { EBDV } \\
\text { TAG }\end{array}$} & \multirow[t]{2}{*}{ SERVICE } & \multicolumn{2}{|c|}{$\begin{array}{c}\text { TOTAL } \\
\text { VOLUME; }\end{array}$} & \multirow{2}{*}{\begin{tabular}{|l|} 
Initi \\
1 \\
Pres \\
- \\
Bars
\end{tabular}} & \multirow{2}{*}{\begin{tabular}{c|} 
Initial \\
Temp \\
C
\end{tabular}} & \multirow{2}{*}{$\begin{array}{r}\text { Final } \\
\text { Pressi } \\
\text { re } \\
\text { Barg }\end{array}$} & \multirow{2}{*}{$\begin{array}{l}\begin{array}{l}\text { Orifice } \\
\text { Area/Diam } \\
\text { eter }\end{array} \\
\mathrm{mm}^{2} / \mathrm{mm}\end{array}$} & \multirow{2}{*}{$\begin{array}{c}\text { Blowdown } \\
\text { Rate } \\
\text { Kg/hr. }\end{array}$} \\
\hline & & Gas & Liq. & & & & & \\
\hline \multirow{2}{*}{$\begin{array}{l}30-B D V- \\
031\end{array}$} & \multirow{2}{*}{$\begin{array}{l}\text { FROM FLOW LINES } \\
\text { THROUGH } \\
\text { PRODUCTION } \\
\text { HEADER TO AIR } \\
\text { COOLERS INLET } \\
\end{array}$} & \multicolumn{2}{|c|}{33.5851} & 131 & 110 & 7 & $\begin{array}{c}762.2 / \\
31.15\end{array}$ & 43300 \\
\hline & & $\begin{array}{l}26.81 \\
6\end{array}$ & 6.7691 & & & & & \\
\hline \multirow{2}{*}{$\begin{array}{l}30-B D V- \\
032\end{array}$} & \multirow{2}{*}{$\begin{array}{l}\text { FROM FLOW LINES } \\
\text { THROUGH TEST } \\
\text { HEADER TO TEST }\end{array}$} & \multicolumn{2}{|c|}{2.2784} & 131 & 90 & 7 & $60 / 8.73$ & 3581 \\
\hline & & 2.21 & 0.0684 & & & & & \\
\hline \multirow{2}{*}{$\begin{array}{l}30-\mathrm{BDV}- \\
033\end{array}$} & \multirow{2}{*}{$\begin{array}{l}\text { AIR COOLERS WITH } \\
\text { INLET /OUTLET AND } \\
\text { BYPASS LINE }\end{array}$} & \multicolumn{2}{|c|}{11.7} & 131 & 92.3 & 7 & $279.3 /$ & 16574 \\
\hline & & 11 & 0.7 & & & & & \\
\hline \multirow{2}{*}{$\begin{array}{l}30-B D V- \\
034\end{array}$} & \multirow{2}{*}{$\begin{array}{l}\text { AIR COOLERS WITH } \\
\text { INLET /OUTLET AND } \\
\text { BYPASS IINE }\end{array}$} & \multicolumn{2}{|c|}{8.1} & 131 & 58 & 7 & $208.5 /$ & 13750 \\
\hline & & 7.6 & 0.5 & & & & & \\
\hline \multirow{2}{*}{$\begin{array}{l}30-B D V- \\
035\end{array}$} & \multirow{2}{*}{$\begin{array}{l}\text { INTERCONNECTING } \\
\text { PIPING BETWEEN } \\
\text { AIR COOLERS \& } \\
\text { PRODUCTION } \\
\text { SEPARATORS } \\
\end{array}$} & \multicolumn{2}{|c|}{17.63} & 131 & 57.8 & 7 & $\begin{array}{l}441.7 / \\
23.71\end{array}$ & 29234 \\
\hline & & 16.51 & 1.12 & & & & & \\
\hline \multirow{2}{*}{$\begin{array}{l}30-\mathrm{BDV}- \\
602\end{array}$} & \multirow{2}{*}{ Production Separator A } & \multicolumn{2}{|c|}{36.82} & 131 & 57.8 & 7 & $883.9 /$ & 56846 \\
\hline & & 29.456 & 7.364 & & & & & \\
\hline \multirow{2}{*}{$\begin{array}{l}30-B D V- \\
702\end{array}$} & \multirow[b]{2}{*}{ Production Separator B } & \multicolumn{2}{|c|}{36.82} & 131 & 57.8 & 7 & $883.9 /$ & 56846 \\
\hline & & 29.456 & 7.364 & & & & & \\
\hline \multirow{2}{*}{$\begin{array}{l}30-\mathrm{BDV}- \\
802\end{array}$} & \multirow[b]{2}{*}{ Production Separator C } & \multicolumn{2}{|c|}{36.82} & 131 & 57.8 & 7 & $883.9 /$ & 56846 \\
\hline & & 29.456 & 7.364 & & & & & \\
\hline \multirow{2}{*}{$\begin{array}{l}30-B D V- \\
505\end{array}$} & & 9 & .1 & 131 & 57.8 & 7 & $226.5 /$ & 14553 \\
\hline & Test Separator & 7.8 & 1.3 & & & & & \\
\hline & & & .23 & 131 & 57.8 & 7 & $448.2 /$ & 28825 \\
\hline 038 & Process gas outlet piping & 18.23 & 0 & & & & & \\
\hline & & & 26 & 131 & 57.8 & 7 & $632.1 /$ & 40653 \\
\hline
\end{tabular}




\begin{tabular}{|c|c|c|c|c|c|c|c|c|}
\hline \multirow{2}{*}{$\begin{array}{r}\text { EBDV } \\
\text { TAG }\end{array}$} & \multirow[t]{2}{*}{ SERVICE } & \multicolumn{2}{|c|}{$\begin{array}{c}\text { TOTAL } \\
\text { VOLUME; }\end{array}$} & \multirow{2}{*}{\begin{tabular}{|l|}
$\mid$ Initi \\
I \\
Pres \\
. \\
Barg \\
\end{tabular}} & \multirow{2}{*}{$\begin{array}{c}\text { Initial } \\
\text { Temp } \\
\mathrm{C}\end{array}$} & \multirow{2}{*}{$\begin{array}{c}\text { Final } \\
\text { Presst } \\
\text { re } \\
\text { Barg }\end{array}$} & \multirow{2}{*}{$\begin{array}{l}\begin{array}{l}\text { Orifice } \\
\text { Area/Diam } \\
\text { eter }\end{array} \\
\mathrm{mm}^{2} / \mathrm{mm}\end{array}$} & \multirow{2}{*}{$\begin{array}{c}\begin{array}{c}\text { Blowdown } \\
\text { Rate }\end{array} \\
\mathrm{Kg} / \mathrm{hr} .\end{array}$} \\
\hline & & Gas & Liq. & & & & & \\
\hline $\begin{array}{l}30-\mathrm{BDV}- \\
037\end{array}$ & $\begin{array}{l}\text { Process gas outlet } \\
\text { piping }\end{array}$ & 25.995 & 0.005 & & & & & \\
\hline \multirow{2}{*}{$\begin{array}{l}\text { 80-BDV- } \\
012\end{array}$} & \multirow{2}{*}{$\begin{array}{l}\text { Inter-piping between } \\
\text { Comp. Discharge \& tie- } \\
\text { in }\end{array}$} & \multicolumn{2}{|c|}{36.304} & 131 & 57.8 & 7 & $\begin{array}{c}873 / \\
33.34 \\
\end{array}$ & 56142 \\
\hline & & 36.304 & 0 & & & & & \\
\hline \multicolumn{8}{|c|}{ TOTAL FLARED GAS DURING BLOWDOWN, Kg/hr. } & 417150 \\
\hline \multicolumn{8}{|c|}{ Flare Designed Flow Rate, $\mathrm{Kg} / \mathrm{hr}$. } & 416800 \\
\hline \multicolumn{8}{|c|}{ Excess of Design \% } & $0 \%$ \\
\hline \multicolumn{8}{|c|}{ Shortage of Design \% } & $-0.08 \%$ \\
\hline
\end{tabular}

Table 2: Total flared gas during blowdown for phase 2 (scenario-1)

\begin{tabular}{|c|c|c|c|c|c|c|c|c|}
\hline \multirow{2}{*}{$\begin{array}{c}\text { EBDV } \\
\text { TAG }\end{array}$} & \multirow[b]{2}{*}{ SERVICE } & \multicolumn{2}{|c|}{ TOTAL } & \multirow{2}{*}{$\begin{array}{l}\text { Initial } \\
\text { Barg }\end{array}$} & \multirow{2}{*}{$\begin{array}{l}\text { Initial } \\
{ }^{\circ} \mathrm{C}\end{array}$} & \multirow{2}{*}{ Final } & \multirow{2}{*}{$\begin{array}{c}\text { Orifice } \\
\underset{\mathrm{m}}{\mathrm{mm}^{2} / \mathrm{m}}\end{array}$} & \multirow{2}{*}{$\begin{array}{c}\text { Blowdow } \\
\mathrm{Kg} / \mathrm{hr} .\end{array}$} \\
\hline & & $\begin{array}{c}\text { Gas } \\
\mathrm{M}^{3}\end{array}$ & $\begin{array}{l}\text { Liq. } \\
\mathrm{M}^{3}\end{array}$ & & & & & \\
\hline \multirow{2}{*}{ 40-BDV-015 } & \multirow{2}{*}{$\begin{array}{l}\text { FROM FLOW LINE } \\
\text { RECEIVER } \\
\text { PACKAGE, }\end{array}$} & \multicolumn{2}{|c|}{33.5851} & 131 & 90 & 7 & $776.4 /$ & 46377 \\
\hline & & \multicolumn{2}{|c|}{26.8166 .7691} & & & & & \\
\hline \multirow{2}{*}{ 40-BDV-016 } & \multirow{2}{*}{$\begin{array}{l}\text { FROM INLET TO AIR } \\
\text { COOLERS, TEST } \\
\text { SEPARATOR, TO } \\
\text { ImAT }\end{array}$} & \multicolumn{2}{|c|}{61.38} & \multirow[t]{2}{*}{131} & \multirow[t]{2}{*}{90} & \multirow[t]{2}{*}{7} & $1403 /$ & \multirow[t]{2}{*}{83798} \\
\hline & & 49.02 & 12.36 & & & & & \\
\hline \multirow{2}{*}{$\begin{array}{c}\text { 40-BDV- } \\
\text { 018A }\end{array}$} & \multirow{2}{*}{$\begin{array}{l}\text { PRODUCTION } \\
\text { SEPARATOR }\end{array}$} & \multicolumn{2}{|c|}{30.69} & \multirow[t]{2}{*}{131} & \multirow[t]{2}{*}{57.8} & \multirow[t]{2}{*}{7} & $\begin{array}{c}742.1 / \\
30.74\end{array}$ & \multirow[t]{2}{*}{47725} \\
\hline & & 15.34 & 15.35 & & & & & \\
\hline \multirow{2}{*}{$\begin{array}{c}\text { 40-BDV- } \\
\text { 018B }\end{array}$} & \multirow{2}{*}{$\begin{array}{l}\text { PRODUCTION } \\
\text { SEPARATOR }\end{array}$} & \multicolumn{2}{|c|}{30.69} & \multirow[t]{2}{*}{131} & \multirow[t]{2}{*}{57.8} & \multirow[t]{2}{*}{7} & $742.1 /$ & \multirow[t]{2}{*}{47725} \\
\hline & & 15.34 & 15.35 & & & & & \\
\hline \multirow{2}{*}{ 40-BDV-004 } & \multirow{2}{*}{$\begin{array}{l}\text { From outlet of } \\
\text { Production Separators to } \\
\text { Phase - } 2 \text { battery limit } \\
\text { ECV at Colom Dinoline }\end{array}$} & \multicolumn{2}{|c|}{14.613} & \multirow[t]{2}{*}{131} & \multirow[t]{2}{*}{57.8} & 7 & $\begin{array}{c}371.3 / \\
21.74\end{array}$ & 24600 \\
\hline & & 14.61 & 0.003 & & & & & \\
\hline RDV 013 & INTERPIPING & 47.6 & 795 & 131 & 57.8 & 7 & $1137 / 38$ & 73139 \\
\hline (new valve) & DISCHARGE \& TIE-IN & 47.67 & 0.0095 & & & & & \\
\hline
\end{tabular}




\begin{tabular}{|c|c|c|c|c|c|c|c|c|}
\hline \multirow{2}{*}{$\begin{array}{c}\text { 80-BDV-014 } \\
\text { (new valve) }\end{array}$} & \multirow{2}{*}{$\begin{array}{l}\text { INTERPIPING } \\
\text { BETWEEN phase-2 -2 } \\
\& \text { TIE-IN }\end{array}$} & \multicolumn{2}{|c|}{15.122} & \multirow[t]{2}{*}{131} & \multirow[t]{2}{*}{57.8} & \multirow[t]{2}{*}{7} & \multirow[t]{2}{*}{$\begin{array}{l}375.6 / \\
21.87\end{array}$} & \multirow[t]{2}{*}{24154} \\
\hline & & 15 & 0.122 & & & & & \\
\hline \multicolumn{8}{|c|}{ TOTAL FLARED GAS DURING BLOWDOWN, Kg/hr. } & 34751 \\
\hline \multicolumn{8}{|c|}{ Flare Designed Flow Rate, $\mathrm{Kg} / \mathrm{hr}$. } & 416800 \\
\hline \multicolumn{8}{|c|}{ Excess of Design \% } & $17 \%$ \\
\hline \multicolumn{8}{|c|}{ Shortage of Design \% } & $0.00 \%$ \\
\hline
\end{tabular}

The compression initial pressure assumed to be the maximum operating pressure for all equipment, as a result of pressure reduction at the compression suction header, the depressuring pressure will vary according to the operating condition. The total peak flow calculated as described in Table 3:

Table 3: Total flared gas during blowdown for the new compression unit

\begin{tabular}{|c|c|c|c|c|c|c|c|c|}
\hline \multirow{3}{*}{$\begin{array}{l}\text { EBDV } \\
\text { TAG }\end{array}$} & \multirow{3}{*}{ SERVICE } & \multicolumn{2}{|c|}{ TOTAL } & \multirow{2}{*}{$\begin{array}{l}\text { Initial } \\
\text { Press. }\end{array}$} & \multirow{2}{*}{$\begin{array}{l}\text { Initial } \\
\text { Temp. }\end{array}$} & \multirow{2}{*}{$\begin{array}{c}\text { Final } \\
\text { Pressure }\end{array}$} & \multirow{2}{*}{$\begin{array}{l}\text { Orifice } \\
\text { Area / } \\
\text { Diam. }\end{array}$} & \multirow{2}{*}{$\begin{array}{l}\text { Blowdo } \\
\text { wn } \\
\text { Rate }\end{array}$} \\
\hline & & \multirow{2}{*}{$\begin{array}{l}\text { Gas } \\
\mathrm{M}^{3}\end{array}$} & \multirow{2}{*}{$\begin{array}{l}\text { Liq. } \\
\mathrm{M}^{3}\end{array}$} & & & & & \\
\hline & & & & Baro & ${ }^{\circ} \mathrm{C}$ & Baro & $\mathrm{mm}^{2} / \mathrm{mm}$ & $\mathrm{Ko} / \mathrm{hr}$ \\
\hline \multirow{2}{*}{$\begin{array}{l}80-\mathrm{BDV}- \\
010\end{array}$} & \multirow{2}{*}{$\begin{array}{l}\text { SEPARATORS } \\
\text { OUTLET TO } \\
\text { COMPRESSION }\end{array}$} & \multicolumn{2}{|c|}{123.865} & \multirow{2}{*}{85} & \multirow{2}{*}{47.58} & \multirow{2}{*}{7} & \multirow{2}{*}{$\begin{array}{c}2457 / \\
55.9\end{array}$} & \multirow{2}{*}{101223} \\
\hline & & 123.86 & 0 & & & & & \\
\hline \multirow{2}{*}{$\begin{array}{l}80-B D V- \\
101\end{array}$} & \multirow{2}{*}{$\begin{array}{l}\text { COMPRESSION } \\
\text { MACHINE TRAIN A }\end{array}$} & \multicolumn{2}{|c|}{62.3} & \multirow{2}{*}{95} & \multirow{2}{*}{72.76} & \multirow{2}{*}{7} & \multirow{2}{*}{$\begin{array}{l}1229 / \\
39.56\end{array}$} & \multirow{2}{*}{52350} \\
\hline & & 57.8 & 4.4747 & & & & & \\
\hline \multirow{2}{*}{$\begin{array}{l}80-B D V- \\
201\end{array}$} & \multirow{2}{*}{$\begin{array}{l}\text { COMPRESSION } \\
\text { MACHINE TRAIN B }\end{array}$} & \multicolumn{2}{|c|}{47.3951} & \multirow{2}{*}{100.23} & \multirow{2}{*}{76.71} & \multirow[b]{2}{*}{7} & \multirow{2}{*}{$\begin{array}{c}956.4 / \\
34.89\end{array}$} & \multirow{2}{*}{42646} \\
\hline & & 44.55 & 2.8451 & & & & & \\
\hline \multirow{2}{*}{$\begin{array}{l}80-\mathrm{BDV}- \\
301\end{array}$} & COMPRESSION & 62.2 & 747 & 05 & 7276 & 7 & $1229 /$ & 52350 \\
\hline & MACHINE TRAIN C & 57.8 & 4.4747 & 95 & 12.10 & 1 & 39.56 & 52550 \\
\hline 80-BDV_- & COMPRESSION & 47.3 & 951 & & & & 95641 & \\
\hline 401 & MACHINE TRAIN D & 44.55 & 2.8451 & 100.23 & 76.71 & 7 & 34.89 & 42646 \\
\hline 80-BDV- & $\begin{array}{l}\text { COMPRESSION } \\
\text { DISCHARGE TO }\end{array}$ & 5 & 8 & & & 7 & $1370 /$ & \\
\hline & FACITILIES & 58 & 0 & & & & & \\
\hline 80-BDV- & CONDENSATE & 28. & 165 & 70 & 578 & 7 & $511.8 /$ & 81065 \\
\hline $001 \mathrm{~A}$ & SUCTION DRUM A & 19.595 & 8.57 & & & & 25.53 & \\
\hline 80-BDV- & CONDENSATE & 28. & 194 & 70 & 578 & 7 & $511.8 /$ & 1065 \\
\hline 001B & SUCTION DRUM B & 19.624 & 8.57 & 10 & 31.8 & 1 & 25.53 & 8190 \\
\hline
\end{tabular}




\begin{tabular}{|c|c|c|c|c|c|c|c|c|}
\hline \multirow{3}{*}{$\begin{array}{l}\text { EBDV } \\
\text { TAG }\end{array}$} & \multirow{3}{*}{ SERVICE } & \multicolumn{2}{|c|}{ TOTAL } & \multirow{2}{*}{$\begin{array}{l}\text { Initial } \\
\text { Press. }\end{array}$} & \multirow{2}{*}{$\begin{array}{l}\text { Initial } \\
\text { Temp. }\end{array}$} & \multirow{2}{*}{$\begin{array}{l}\text { Final } \\
\text { Pressure }\end{array}$} & \multirow{2}{*}{$\begin{array}{l}\text { Orifice } \\
\text { Area / } \\
\text { Diam. }\end{array}$} & \multirow{2}{*}{$\begin{array}{c}\text { Blowdo } \\
\text { wn } \\
\text { Rate }\end{array}$} \\
\hline & & \multirow{2}{*}{$\begin{array}{l}\text { Gas } \\
\mathrm{M}^{3}\end{array}$} & \multirow{2}{*}{$\begin{array}{l}\text { Liq. } \\
\mathrm{M}^{3}\end{array}$} & & & & & \\
\hline & & & & Baro & ${ }^{\circ} \mathrm{C}$ & Baro & $\mathrm{mm}^{2} / \mathrm{mm}$ & $\mathrm{Ko} / \mathrm{hr}$ \\
\hline \multirow{2}{*}{$\begin{array}{l}80-B D V- \\
002\end{array}$} & \multirow{2}{*}{$\begin{array}{l}\text { FLASH GAS } \\
\text { PREHEATER }\end{array}$} & \multicolumn{2}{|c|}{0.068} & \multirow{2}{*}{70} & \multirow{2}{*}{57.8} & \multirow{2}{*}{7} & \multirow{2}{*}{$\begin{array}{c}1.264 / \\
1.269\end{array}$} & \multirow{2}{*}{40.49} \\
\hline & & 0.068 & 0 & & & & & \\
\hline \multirow{2}{*}{$\begin{array}{l}\text { 80-BDV- } \\
004 \mathrm{~A}\end{array}$} & \multirow{2}{*}{$\begin{array}{l}\text { HP FUEL GAS } \\
\text { PREHEATER }\end{array}$} & \multicolumn{2}{|c|}{2.304} & \multirow{2}{*}{130} & \multirow{2}{*}{57.44} & \multirow{2}{*}{7} & \multirow{2}{*}{$\begin{array}{c}59.15 / \\
8.67\end{array}$} & \multirow{2}{*}{1889} \\
\hline & & 2.304 & 0 & & & & & \\
\hline \multirow{2}{*}{$\begin{array}{l}\text { 80-BDV- } \\
004 \mathrm{~B}\end{array}$} & \multirow{2}{*}{$\begin{array}{l}\text { HP FUEL GAS } \\
\text { PREHEATER }\end{array}$} & \multicolumn{2}{|c|}{2.258} & \multirow{2}{*}{130} & \multirow{2}{*}{57.44} & \multirow{2}{*}{7} & \multirow{2}{*}{$\begin{array}{c}59.15 / \\
8.67\end{array}$} & \multirow{2}{*}{1889} \\
\hline & & 2.258 & 0 & & & & & \\
\hline \multirow{2}{*}{$\begin{array}{l}80-B D V- \\
006\end{array}$} & \multirow{2}{*}{$\begin{array}{l}\text { HP FUEL GAS KO } \\
\text { DRUM }\end{array}$} & \multicolumn{2}{|c|}{7.291} & & & & $103.3 /$ & \\
\hline & & 7.291 & 0 & 42 & 50 & 7 & 11.47 & 1935 \\
\hline TOTAL FI & RED GAS DURING & WDOV & $\mathrm{N}, \mathrm{Kg} /$ & & & & & $\begin{array}{c}400738.4 \\
9\end{array}$ \\
\hline Flare Desi & ed Flow Rate, $\mathrm{Kg} / \mathrm{hr}$ & & & & & & & 416800 \\
\hline Excess of I & $\operatorname{sign} \%$ & & & & & & & $4 \%$ \\
\hline Shortage o & esign $\%$ & & & & & & & $0.00 \%$ \\
\hline
\end{tabular}

The results above show that the total flared gas directed to phase-1 flare will be $417150 \mathrm{Kg} / \mathrm{hr}$. (it exceeds the design flare capacity by $0.08 \%$ of design capacity $416800 \mathrm{Kg} / \mathrm{hr}$.) which is accepted considering the flare design margin. The total flared gas directed to phase-2 flare will be 347518 $\mathrm{Kg} / \mathrm{hr}$. (which is lesser than the design flare capacity by $17 \%$ of design capacity 416800 $\mathrm{Kg} / \mathrm{hr}$.). The total flared gas directed to an identical new flare will be $400738 \mathrm{Kg} / \mathrm{hr}$. (which is lesser than the design flare capacity by $4 \%$ ). The new identical flare system estimated cost with $+/-10 \%$ accuracy is illustrated in Table 4.

Table 4: New Identical Flare System Cost Estimation

\begin{tabular}{|l|l|}
\hline \multicolumn{1}{|c|}{ Equipment } & \multicolumn{1}{c|}{ Cost (\$) } \\
\hline 200 m Piping (16" Duplex): Headers, sub headers and supports & 400,000 \\
\hline Carbon Steel HP Flare Knock Out Drum & $1,000,000$ \\
\hline Carbon Steel HP Flare Stack & $1,000,000$ \\
\hline Total & $2,400,000$ \\
\hline
\end{tabular}

Adding a new independent compression flare utility will positively help relieving the new compression surge volume in a minimum time as per API521 standard (15 minutes or less). Independent flare will not affect existing facilities depressuring time and in addition the project will not be affected when one of the existing flares is under maintenance. But on the other hand, will negatively affect the project budget as this requires a high capital cost of the new flare system. 
Scenario-2: Sequenced Flare to Phase-1 and Phase-2 existing

Simultaneously depressurizing all the process and equipment, vessels, and piping in a plant will be difficult to accomplish (due to pipe sizing and economic impacts). In these cases, sequential or segmented depressurization of vessels should be considered. Providing for the worst vessels first or controlling the system to depressurize the area most affected first are possible scenarios that can be employed. Sequential blowdown starts from equipment maximum operating pressure to 7 Barg within 15 minutes. This scenario is performed in order to estimate the peak blowdown rate for each zone based on external fire depressuring criteria and to depressurize to 7 Barg in 15 minutes:

The results of scenario 2 show that:

1- Once the compression facility starts to relief to phase-1 flare after phase-1 piping and equipment have reached to $7 \mathrm{barg}$ at $15 \mathrm{~min}$, the total flared gas will be handled by phase1 flare for the following 15 minutes is $418812.49 \mathrm{Kg} / \mathrm{hr}$ (Table 5). (which exceeds with accepted limits the design flare capacity of PH-1 by $0.5 \%$ of flare design capacity $416800 \mathrm{Kg} / \mathrm{hr}$.). That means that the sequential blowdown solution will not meet the phase-1 flare design requirements.

2- Once the compression facility starts to relief to phase-2 flare, after Phase-2 piping and equipment have reached to 7 barg at $15 \mathrm{~min}$, the total flared gas will be handled by phase2 flare for the following $15 \mathrm{~min}$. is 415604.49 $\mathrm{Kg} / \mathrm{hr}$ (Table 6). (which is lesser than the design flare capacity by $0.3 \%$ of flare design capacity $416800 \mathrm{Kg} / \mathrm{hr}$.). That means that the sequential blowdown solution will meet the phase-2 flare design requirements and also the blowdown criteria.

3- Adding the new compression facility considering the sequenced flaring technique will cost a new system of uninterrupted power supply (UPS) with logic solver to resume efficient in case of total plant power failure. Use of UPS or emergency diesel generator may be considered so that when the normal power supply is interrupted, a standby power supply would automatically start, in a fraction of a second, to support critical equipment or units. Estimated cost with +/$10 \%$ accuracy for new redundant UPS system to avoid any failure causing direct flaring at the same time is approximately $10000 \$$.

Table 5: Total Flared Gas during Blowdown for phase-1 (scenario- 2)

\begin{tabular}{|c|c|c|c|c|c|c|c|c|c|}
\hline \multirow{3}{*}{$\begin{array}{l}\text { EBDV } \\
\text { TAG }\end{array}$} & \multirow{3}{*}{ SERVICE } & \multicolumn{2}{|c|}{ TOTAL } & \multirow{2}{*}{$\begin{array}{l}\text { Initial } \\
\text { Press. }\end{array}$} & \multirow{2}{*}{$\begin{array}{l}\text { Initial } \\
\text { Temp }\end{array}$} & \multirow{2}{*}{$\begin{array}{l}\text { Fin } \\
\text { al }\end{array}$} & \multirow{2}{*}{$\begin{array}{c}\text { Orifice } \\
\text { Area/Diam }\end{array}$} & \multirow{2}{*}{$\begin{array}{c}\text { Blowdo } \\
\text { wn } \\
\end{array}$} & \multirow{2}{*}{$\begin{array}{l}\text { Balanc } \\
\text { e Flow }\end{array}$} \\
\hline & & \multirow{2}{*}{$\begin{array}{c}\text { GAS } \\
\mathrm{M}^{3}\end{array}$} & \multirow{2}{*}{$\begin{array}{l}\text { LIQ. } \\
\mathrm{M}^{3}\end{array}$} & & & & & & \\
\hline & & & & Barg & $\mathrm{C}$ & $\begin{array}{c}\text { Bar } \\
\mathrm{g}\end{array}$ & $\mathrm{mm} 2 / \mathrm{mm}$ & $\mathrm{Kg} / \mathrm{hr}$ & \\
\hline \multirow[b]{2}{*}{$\begin{array}{c}\text { 30-BDV- } \\
031\end{array}$} & \multirow{2}{*}{$\begin{array}{c}\text { FROM FLOW LINES } \\
\text { THROUGH } \\
\text { PRODUCTION } \\
\text { HEADER TO AIR } \\
\text { COOI ER INI ET } \\
\end{array}$} & \multicolumn{2}{|c|}{33.5851} & \multirow[b]{2}{*}{131} & \multirow[b]{2}{*}{110} & \multirow[b]{2}{*}{7} & \multirow[b]{2}{*}{$\begin{array}{c}762.2 / \\
31.15\end{array}$} & \multirow[b]{2}{*}{43300} & \multirow[b]{2}{*}{1895} \\
\hline & & $\begin{array}{c}26.81 \\
6\end{array}$ & $\begin{array}{c}6.769 \\
1\end{array}$ & & & & & & \\
\hline \multirow{3}{*}{$\begin{array}{c}\text { 30-BDV- } \\
032\end{array}$} & \multirow{2}{*}{$\begin{array}{c}\text { FROM FLOW LINES } \\
\text { THROUGH TEST } \\
\text { HEADER TO TEST } \\
\text { SEPARATOR INLET }\end{array}$} & \multicolumn{2}{|c|}{2.2784} & \multirow[b]{2}{*}{131} & \multirow[b]{2}{*}{90} & \multirow[b]{2}{*}{7} & \multirow[b]{2}{*}{$60 / 8.73$} & \multirow[b]{2}{*}{3581} & \multirow[b]{2}{*}{144.6} \\
\hline & & 2.21 & $\begin{array}{c}0.068 \\
4\end{array}$ & & & & & & \\
\hline & & \multicolumn{2}{|c|}{11.7} & 131 & 92.3 & 7 & & 16574 & 662.4 \\
\hline
\end{tabular}




\begin{tabular}{|c|c|c|c|c|c|c|c|c|c|}
\hline \multirow{3}{*}{$\begin{array}{c}\text { EBDV } \\
\text { TAG }\end{array}$} & \multirow{3}{*}{ SERVICE } & \multicolumn{2}{|c|}{ TOTAL } & \multirow{2}{*}{$\begin{array}{l}\text { Initial } \\
\text { Press. }\end{array}$} & \multirow{2}{*}{$\begin{array}{l}\text { Initial } \\
\text { Temp }\end{array}$} & \multirow{2}{*}{$\begin{array}{c}\text { Fin } \\
\text { al }\end{array}$} & \multirow{2}{*}{$\begin{array}{c}\text { Orifice } \\
\text { Area/Diam }\end{array}$} & \multirow{2}{*}{$\begin{array}{l}\text { Blowdo } \\
\text { wn }\end{array}$} & \multirow{2}{*}{$\begin{array}{l}\text { Balanc } \\
\text { e Flow }\end{array}$} \\
\hline & & \multirow{2}{*}{$\begin{array}{c}\text { GAS } \\
\mathrm{M}^{3}\end{array}$} & \multirow{2}{*}{$\begin{array}{l}\text { LIQ. } \\
\mathrm{M}^{3}\end{array}$} & & & & & & \\
\hline & & & & Barg & $\mathrm{C}$ & $\begin{array}{c}\text { Bar } \\
\sigma\end{array}$ & $\mathrm{mm} 2 / \mathrm{mm}$ & $\mathrm{Kg} / \mathrm{hr}$ & \\
\hline $\begin{array}{c}\text { 30-BDV- } \\
033\end{array}$ & $\begin{array}{l}\text { AIR COOLERS WITH } \\
\text { INLET /OUTLET AND } \\
\text { BYPASS LINE }\end{array}$ & 11 & 0.7 & & & & $\begin{array}{c}279.3 / \\
18.86\end{array}$ & & \\
\hline \multirow{2}{*}{$\begin{array}{c}\text { 30-BDV- } \\
034\end{array}$} & \multirow{2}{*}{$\begin{array}{c}\text { AIR COOLERS WITH } \\
\text { INLET /OUTLET AND } \\
\text { BYPASS LINE }\end{array}$} & \multicolumn{2}{|c|}{8.1} & \multirow[b]{2}{*}{131} & \multirow[b]{2}{*}{58} & \multirow[b]{2}{*}{7} & \multirow{2}{*}{$\begin{array}{c}208.5 / \\
16.29\end{array}$} & \multirow[b]{2}{*}{13750} & \multirow[b]{2}{*}{531.8} \\
\hline & & 7.6 & 0.5 & & & & & & \\
\hline \multirow[b]{2}{*}{$\begin{array}{c}\text { 30-BDV- } \\
035\end{array}$} & \multirow{2}{*}{$\begin{array}{c}\text { INTERCONNECTING } \\
\text { PIPING BETWEEN AIR } \\
\text { COOLERS \& } \\
\text { PRODUCTION } \\
\text { SEPARATORS } \\
\end{array}$} & \multicolumn{2}{|c|}{17.63} & \multirow[b]{2}{*}{131} & \multirow[b]{2}{*}{57.8} & \multirow[b]{2}{*}{7} & \multirow[b]{2}{*}{$\begin{array}{c}441.7 / \\
23.71\end{array}$} & \multirow[b]{2}{*}{29234} & \multirow[b]{2}{*}{1145} \\
\hline & & 16.51 & 1.12 & & & & & & \\
\hline 30-BDV- & Production Senarator A & 36 & & 131 & 578 & 7 & $883.9 /$ & 56846 & 2512 \\
\hline 602 & 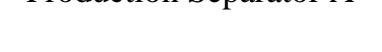 & 29.45 & 7.364 & 101 & 81.0 & $r$ & 33.55 & 30070 & 2012 \\
\hline 30-BDV- & Production Senaretor $\mathrm{B}$ & 36 & & 131 & 578 & 7 & $883.9 /$ & 56846 & 2512 \\
\hline 702 & Fе & 29.45 & 7.364 & 10 & (1.0 & $r$ & 33.55 & 30070 & 2010 \\
\hline 30-BDV- & & 36 & & & & & $883.9 /$ & & \\
\hline 802 & Production Separator C & $\begin{array}{c}29.45 \\
6 \\
\end{array}$ & 7.364 & 131 & 57.8 & 7 & 33.55 & 56846 & 2512 \\
\hline 30-BDV- & & 9 & & 121 & & & $226.5 /$ & 14552 & 6000 \\
\hline 505 & 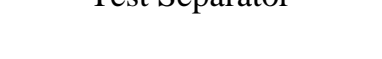 & 7.8 & 1.3 & 101 & (5) & r & 16.9 & $1+000$ & 000.2 \\
\hline 30-BDV- & & 18 & & & & & $448.2 /$ & & \\
\hline 038 & Process gas outlet piping & 18.23 & 0 & 131 & 57.8 & 7 & 23.89 & 28825 & 1245 \\
\hline 30-BDV- & Procecs $\alpha$ as outlet ninino & 2 & & 131 & 578 & 7 & $632.1 /$ & 40653 & 1760 \\
\hline 037 & 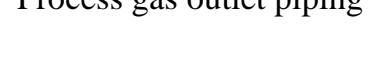 & 25.99 & 0.005 & 101 & 51.0 & $r$ & 28.37 & 40053 & $1 / 09$ \\
\hline 80-BDV- & Inter-piping between & 36. & & & & & & & \\
\hline 012 & $\begin{array}{c}\text { Comp. Discharge \& tie- } \\
\text { in }\end{array}$ & 36.30 & 0 & 131 & $3 / .8$ & 1 & $8 / 3 / 33.34$ & 50142 & 2400 \\
\hline TOTAL FI & ARED GAS DURING BLC & WDOW & $\mathrm{N}, \mathrm{Kg} / \mathrm{h}$ & & & & & 417150 & 18074 \\
\hline Flare Desis & ned Flow Rate, Kg/hr & & & & & & & 416800 & 416800 \\
\hline Excess of I & esign $\%$ & & & & & & & $0 \%$ & \\
\hline Shortage o & Design $\%$ & & & & & & & $-0.08 \%$ & \\
\hline Required C & ompression Flared Gas & & & & & & & & 400738 \\
\hline In Case of & equential Blowdown, Total & Compre & sion Fla & ed Gas & & & & & 418812 \\
\hline In Case of & Sequential Blowdown, Exce & s of Des & gn $\%$ & & & & & & $0.0 \%$ \\
\hline In Case of & equential Blowdown, Shor & ge of D & $\operatorname{sign} \%$ & & & & & & $-0.5 \%$ \\
\hline
\end{tabular}


Table 6: Total Flared Gas during Blowdown for Phase-2 (scenario-2)

\begin{tabular}{|c|c|c|c|c|c|c|c|c|c|}
\hline \multirow{2}{*}{$\begin{array}{l}\text { EBDV } \\
\text { TAG }\end{array}$} & \multirow{2}{*}{ SERVICE } & \multicolumn{2}{|c|}{$\begin{array}{l}\text { TOTAL } \\
\text { VOLUME; }\end{array}$} & $\begin{array}{l}\text { Ini } \\
\text { tial } \\
\text { Pr } \\
\text { ess }\end{array}$ & $\begin{array}{l}\text { Init } \\
\text { ial } \\
\text { Te } \\
\mathrm{mp}\end{array}$ & $\begin{array}{l}\mathrm{Fi} \\
\text { nal } \\
\mathrm{Pr} \\
\text { ess }\end{array}$ & $\begin{array}{l}\text { Orifice } \\
\text { Area/ } \\
\text { Diamet }\end{array}$ & $\begin{array}{l}\text { Blow } \\
\text { down } \\
\text { Rate }\end{array}$ & $\begin{array}{l}\text { Bala } \\
\text { nce } \\
\text { Flow } \\
\text { After }\end{array}$ \\
\hline & & $\begin{array}{c}\text { Gas } \\
\mathrm{m}^{3}\end{array}$ & $\begin{array}{l}\text { Liq. } \\
\mathrm{m}^{3}\end{array}$ & $\begin{array}{l}\mathrm{Ba} \\
\mathrm{rg}\end{array}$ & $\mathrm{C}$ & $\begin{array}{l}\mathrm{Ba} \\
\mathrm{rg}\end{array}$ & $\begin{array}{l}\mathrm{mm}^{2} / \mathrm{m} \\
\mathrm{m}\end{array}$ & $\mathrm{Kg} / \mathrm{hr}$ & $\begin{array}{l}\mathrm{Kg} / \mathrm{h} \\
\text { r. }\end{array}$ \\
\hline \multirow{2}{*}{$\begin{array}{l}40- \\
\text { BDV- } \\
015\end{array}$} & \multirow{2}{*}{\begin{tabular}{lcccc} 
FROM & FLOW & LINE & RECEIVER & \multicolumn{2}{c}{ PACKAGE, } \\
PRODUCTION & HEADER & PACKAGE, & TO AIR \\
COOLERS & & & &
\end{tabular}} & \multicolumn{2}{|c|}{33.56} & 13 & 90 & 7 & $776.4 /$ & 4637 & 1967 \\
\hline & & 26.8 & 6.76 & & & & & & \\
\hline \multirow{2}{*}{$\begin{array}{l}40- \\
\text { BDV- } \\
016\end{array}$} & \multirow{2}{*}{$\begin{array}{l}\text { FROM INLET TO AIR COOLERS, TEST SEPARATOR, } \\
\text { TO INLET OF PRODUCTION SEPARATORS }\end{array}$} & \multicolumn{2}{|c|}{61.38} & $\begin{array}{l}13 \\
1\end{array}$ & 90 & 7 & $\begin{array}{l}1403 / \\
42.26\end{array}$ & $\begin{array}{l}8379 \\
8\end{array}$ & 3523 \\
\hline & & $\begin{array}{c}49.0 \\
2\end{array}$ & $\begin{array}{c}12.3 \\
6\end{array}$ & & & & & & \\
\hline \multirow{2}{*}{$\begin{array}{l}\text { 40- } \\
\text { BDV- } \\
018 \mathrm{~A}\end{array}$} & \multirow{2}{*}{ PRODUCTION SEPARATOR } & \multicolumn{2}{|c|}{30.6} & $\begin{array}{l}13 \\
1\end{array}$ & $\begin{array}{l}57 . \\
8\end{array}$ & 7 & $\begin{array}{c}742.1 / \\
30.74\end{array}$ & $\begin{array}{c}4772 \\
5\end{array}$ & 2086 \\
\hline & & 15.3 & 15.3 & & & & & & \\
\hline \multirow{2}{*}{$\begin{array}{l}40- \\
\text { BDV- } \\
018 B\end{array}$} & \multirow{2}{*}{ PRODUCTION SEPARATOR } & \multicolumn{2}{|c|}{30.6} & 13 & 57. & 7 & $742.1 /$ & 4772 & 2086 \\
\hline & & 15.3 & 15.3 & & & & & & \\
\hline \multirow{2}{*}{$\begin{array}{l}40- \\
\text { BDV- } \\
004\end{array}$} & \multirow{2}{*}{$\begin{array}{l}\text { From outlet of Production Separators to Phase- } 2 \text { battery } \\
\text { limit ESV at Salam Pipeline }\end{array}$} & \multicolumn{2}{|c|}{14.603} & $\begin{array}{l}13 \\
1\end{array}$ & $\begin{array}{l}57 . \\
8\end{array}$ & 7 & $\begin{array}{l}371.3 / \\
21.74\end{array}$ & $\begin{array}{c}2460 \\
0\end{array}$ & 968 \\
\hline & & 14.6 & $\begin{array}{c}0.00 \\
3\end{array}$ & & & & & & \\
\hline \multirow{2}{*}{$\begin{array}{l}80- \\
\text { BDV- } \\
013 \\
\text { (NEW } \\
\text { VALV } \\
\text { E) }\end{array}$} & \multirow[b]{2}{*}{$\begin{array}{l}\text { INTERPIPING BETWEEN COMP. DISCHARGE \& TIE- } \\
\text { IN }\end{array}$} & \multicolumn{2}{|c|}{47.609} & $\begin{array}{l}13 \\
1\end{array}$ & $\begin{array}{l}57 . \\
8\end{array}$ & 7 & $\begin{array}{c}1137 / \\
38\end{array}$ & $\begin{array}{c}7313 \\
9\end{array}$ & 3221 \\
\hline & & 47.6 & $\begin{array}{c}0.00 \\
9\end{array}$ & & & & & & \\
\hline \multirow{2}{*}{$\begin{array}{l}80- \\
\text { BDV- } \\
014 \\
\text { (NEW } \\
\text { VALV } \\
\text { E) }\end{array}$} & \multirow[b]{2}{*}{ INTERPIPING BETWEEN PH II \& TIE-IN } & \multicolumn{2}{|c|}{15.12} & $\begin{array}{l}13 \\
1\end{array}$ & $\begin{array}{l}57 . \\
8\end{array}$ & 7 & $\begin{array}{c}375.6 / \\
21.87\end{array}$ & $\begin{array}{c}2415 \\
4\end{array}$ & 1015 \\
\hline & & 15 & 0.12 & & & & & & \\
\hline \multicolumn{8}{|c|}{ TOTAL FLARED GAS DURING BLOWDOWN, Kg/hr } & 3475 & 1486 \\
\hline \multicolumn{8}{|c|}{ Flare Designed Flow Rate, $\mathrm{Kg} / \mathrm{hr}$} & 4168 & 4168 \\
\hline \multicolumn{8}{|c|}{ Excess of Design \% } & $17 \%$ & \\
\hline \multicolumn{8}{|c|}{ Shortage of Design \% } & 0.00 & \\
\hline \multicolumn{8}{|c|}{ Required Compression Flared Gas } & & 4007 \\
\hline
\end{tabular}




\begin{tabular}{|c|c|c|c|c|c|c|c|c|c|}
\hline \multirow{2}{*}{$\begin{array}{l}\text { EBDV } \\
\text { TAG }\end{array}$} & \multirow{2}{*}{ SERVICE } & \multicolumn{2}{|c|}{$\begin{array}{c}\text { TOTAL } \\
\text { VOLUME; }\end{array}$} & \multirow{2}{*}{$\begin{array}{l}\text { Ini } \\
\text { tial } \\
\mathrm{Pr} \\
\text { ess } \\
\cdot \\
\mathrm{Ba} \\
\mathrm{rg}\end{array}$} & \multirow{2}{*}{$\begin{array}{l}\text { Init } \\
\text { ial } \\
\mathrm{Te} \\
\mathrm{mp} \\
\cdot \\
\mathrm{C}\end{array}$} & \multirow{2}{*}{$\begin{array}{l}\mathrm{Fi} \\
\mathrm{nal} \\
\mathrm{Pr} \\
\text { ess } \\
\cdot \\
\mathrm{Ba} \\
\mathrm{rg}\end{array}$} & \multirow{2}{*}{$\begin{array}{l}\text { Orifice } \\
\text { Area/ } \\
\text { Diamet } \\
\text { er } \\
\\
\mathrm{mm}^{2} / \mathrm{m} \\
\mathrm{m}\end{array}$} & \multirow{2}{*}{$\begin{array}{l}\text { Blow } \\
\text { down } \\
\text { Rate }\end{array}$} & \multirow{2}{*}{$\begin{array}{l}\text { Bala } \\
\text { nce } \\
\text { Flow } \\
\text { After } \\
15 \\
\text { min } \\
\mathrm{Kg} / \mathrm{h} \\
\mathrm{r} .\end{array}$} \\
\hline & & $\begin{array}{l}\text { Gas } \\
\mathrm{m}^{3}\end{array}$ & $\begin{array}{l}\text { Liq. } \\
\mathrm{m}^{3}\end{array}$ & & & & & & \\
\hline \multicolumn{8}{|c|}{ Total Compression Flared Gas } & & 4156 \\
\hline \multicolumn{8}{|c|}{ In Case of Sequential Blowdown, Excess of Design \% } & & $0.3 \%$ \\
\hline \multicolumn{8}{|c|}{ In Case of Sequential Blowdown, Shortage of Design \% } & & $0.0 \%$ \\
\hline
\end{tabular}

\section{Conclusion}

The two scenarios for connecting a new flare system of the new compression unit to the two existing flare systems are considerd. The two scenarios are implementing a new flare system for the new compression unit and sequential blowdown,. Simulation and calculations revealed that:

For scenario 1, if a new flare system is used (Final Pressure 7 barg within $15 \mathrm{~min}$ ) the total flared gas will be directed to Phase-1 flare will be 417150 $\mathrm{Kg} / \mathrm{hr}$. (it exceeds the design flare capacity by $0.08 \%$ of design capacity $416800 \mathrm{Kg} / \mathrm{hr}$.) which is accepted considering the flare design margin, and the total flared gas will be directed to Phase- 2 flare will be $347518 \mathrm{Kg} / \mathrm{hr}$. (which is lesser than the design flare capacity by $17 \%$ of design capacity $416800 \mathrm{Kg} / \mathrm{hr}$.). For compression depressurization, the total flared gas will be directed to an identical new flare will be $400738 \mathrm{Kg} / \mathrm{hr}$ (which is lesser than the design flare capacity by $4 \%$ ). A new flare for the peak load of $400738 \mathrm{Kg} / \mathrm{hr}$ will require a large capital cost which is approximately 2,400,000 $\$$.

For scenario 2, if sequential blowdown (final Pressure 7 Barg in $15 \mathrm{~min}$.) is used, Once the compression facility starts to relief to Phase-1 flare after Phase-1 piping and equipment have reached to $7 \mathrm{barg}$ at $15 \mathrm{~min}$, the total flared gas will be handled by Phase-1 flare for the following $15 \mathrm{~min}$. is $418812.49 \mathrm{Kg} / \mathrm{hr}$. (which exceeds the design flare capacity by $0.5 \%$ of flare design capacity $416800 \mathrm{Kg} / \mathrm{hr}$. which is accepted considering the flare design margin). Once the compression facility starts to relief to Phase-2 flare, after Phase-2 piping and equipment have reached to $7 \mathrm{barg}$ at $15 \mathrm{~min}$, the total flared gas will be handled by Phase- 2 flare for the following $15 \mathrm{~min}$. is $415604.49 \mathrm{Kg} / \mathrm{hr}$. (which is lesser than the design flare capacity by $0.3 \%$ of flare design capacity $416800 \mathrm{Kg} / \mathrm{hr}$. Adding the new compression facility considering the sequenced flaring technique will cost a new system of UPS with logic solver to resume efficient in case of total plant power failure, it approximately costs 10000 \$.

\section{References}

[1] Karl Kolmetz, Riska Ristiyanti, Kolmetz Handbook of Process Equipment Design FLARE SYSTEMS SAFETY, SELECTION AND SIZING AND TROUBLESHOOTING (ENGINEERING DESIGN GUIDELINE), KLM Technology Group Practical Engineering Guidelines for Processing Plant Solutions, 2015

[2] Carbon Limits AS., Flare Reduction in Egypt report, 15.04.2016.

[3] Mikhail Zhizhin, Kimberly Baugh, FengChi Hsu CIRES, Results from a Survey of Global Natural Gas Flaring from Visible Infrared Imaging Radiometer Suite Data, Chris Elvidge, Earth observation group, NOAA National Centers for Environmental Information, May 18, 2016.

[4] ADAM BADER, CHARLES E.BAUKAL, JR., P.E., WES BUSSMAN, Selecting the 
proper flare system, American Institute of Chemical Engineers, 2011

[5] Daniel A. Crowl, Joseph F. Louvar, Chemical Process Safety, Fundamentals with Applications, $3^{\text {rd }}$ edition ,2011

[6] Douglas Attawy, David A. Coyle, GROUND FLARES - OUT OF SIGHT, OUT OF MIND. LNG Industry, PERTH AUSTRALIA, 11-15 April 2016.

[7] Ken Arnold, Maurice Stewart, Surface Production Operations, Volume 2: 2nd Edition, Design of Gas-Handling Systems and Facilities, Gulf Publishing Company, 1999.

[8] Nicholas P. Cheremisinoff, Industrial Gas Flaring Practices, PhD, John Wiley \& Sons Inc, 2013.

[9] Pressure-relieving and depressuring systems, ANSI/API STANDARD 521, FIFTH EDITION, JANUARY 2007 (INCLUDES ERRATA JUNE 2007).

[10] Frank Lees, Lee's Loss Prevention in the Process Industries, 3rd Edition, Volume 1, Butterworth-Heinemann, 2005.

[11] Flare Details for General Refinery and Petrochemical Service, ANSI/API STANDARD 537, SECOND EDITION, DECEMBER 2008.

[12] Don W. Green, Robert H. Perry, Perry's Chemical Engineers' Handbook, $8^{\text {th }}$ Edition, McGraw-Hill Companies, Inc., 2008

[13] Dennis P. Nolan, Handbook of Fire and Explosion Protection Engineering Principles: for Oil, Gas, Chemical and Related Facilities, 4th edition, Gulf Professional Publishing 2019.

[14] Charles E. Baukal Jr., The John Zink Hamworthy Combustion Handbook : Fundamentals (Industrial Combustion), Volume 1, 2nd edition, CRC Press, 2012 